\title{
Big data analytics of crime prevention and control based on image processing upon cloud computing
}

\author{
Zheng $\mathrm{Xu}^{1}$, Cheng Cheng ${ }^{1,2}$, Vijayan Sugumaran ${ }^{3}$ \\ 'School of Computer Science, Shanghai University, Shanghai 201142, China. \\ ${ }^{2}$ Cernter of IoT, The third research institute of the ministry of public security, Shanghai 200335, China. \\ ${ }^{3}$ Department of Decision and Information Sciences, Oakland University, Rochester, MI 48309, USA.
}

Correspondence to: Prof. Zheng Xu, School of Computer Science, Shanghai University, Shanghai 201142, China.

E-mail: zhengxu@shu.edu.cn

How to cite this article: Xu Z, Cheng C, Sugumaran V. Big data analytics of crime prevention and control based on image processing upon cloud computing. J Surveil/ Secur Saf2020;1:16-33. http://dx.doi.org/10.20517/jsss.2020.04

Received: 5 Mar 2020 First Decision: 21 Apr 2020 Revised: 5 Jun 2020 Accepted: 12 Aug 2020 Available online: 12 Sep 2020

Academic Editor: Yelena Yesha Copy Editor: Cai-Hong Wang Production Editor: Jing Yu

\begin{abstract}
Aim: Current crime behavior observation has the problem of not being real time, thus criminal behavior cannot be promptly controlled. To improve the control of criminal behavior, this study was based on cloud computing image processing, and adopted data mining for criminal behavior.
\end{abstract}

Methods: This study obtained many criminal behavior characteristics through data collection and combined the rapid response capability of cloud computing to adopt data processing. In addition, to improve the accuracy of criminal behavior recognition, the identification method for criminal behaviors in selected populations was studied, and the image processing technology was combined to identify individual crimes and subject segmentation.

Results: Our work used statistical methods to collect the characteristics of criminal behavior, and we designed experiments to verify the effectiveness of the algorithm. The experimental research shows that the algorithm has high accuracy in identifying abnormal behavior.

Conclusion: The research shows that the accuracy of the algorithm for identifying abnormal behavior is relatively high, and it has high practical value, which can meet the accuracy and real-time requirements of security systems.

Keywords: Cloud computing, cloud storage, data mining, crime prevention and control, image capture

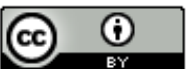

(C) The Author(s) 2020. Open Access This article is licensed under a Creative Commons Attribution 4.0 International License (https://creativecommons.org/licenses/by/4.0/), which permits unrestricted use, sharing, adaptation, distribution and reproduction in any medium or format, for any purpose, even commercially, as long as you give appropriate credit to the original author(s) and the source, provide a link to the Creative Commons license, and indicate if changes were made. 


\section{INTRODUCTION}

Current crime behavior observation has the problem of not being real time, thus criminal behavior cannot be promptly controlled. To improve the control of criminal behavior, this study was based on cloud computing image processing, and adopted data mining for criminal behavior. his study obtained many criminal behavior characteristics through data collection and combined the rapid response capability of cloud computing to adopt data processing.

Criminal behavior has always affected the stability of societies, and it is difficult to control crimes in real time through monitoring and observation. In recent years, with the continuous advancement of society, the level of material and spiritual living has continuously improved, and people have begun to pay more attention to the safety of their lives and property. As a settlement of the population, the city has a more urgent need to ensure that life and property are not invaded. Due to the high population density and complex personnel structure, urban management becomes more and more difficult, and various public security incidents are more likely to occur. How to effectively control crimes is an important research content of current social management, and the analysis of big data crime behavior is an effective way.

The abnormality detection object in this paper is a random group in ordinary public places, i.e., the people in the crowd are not unified and purposeful. The direction of movement of the crowd is irregular when there is no abnormality. This paper uses the anomaly detection algorithm with improved acceleration characteristics to detect the abnormal escape behavior of the crowd. Firstly, the motion vector field is processed by block processing, then the image is filtered to reduce the influence of noise, and the mean filtering is adopted, and then the algorithm is used to extract the foreground of the image sequence. This kind of operation not only facilitates the extraction of motion features, but also reduces the disadvantages of large computational complexity. The experimental research shows that the algorithm has high accuracy in identifying abnormal behavior and has high practical value, which can meet the accuracy and real-time requirements of the security system.

\section{RELATED WORK}

In recent years, with the continuous deepening of the research on abnormal behavior detection of people, related technologies have gradually matured. According to the degree of occurrence of abnormal events in the surveillance video picture, they can be divided into global abnormalities and local abnormalities. A global exception means that an abnormal behavior occurs in the entire monitored image (even if part of the area is normal). A local anomaly is an abnormal behavior in which a local area in a surveillance video is distinguished from a surrounding area. In addition, the focus of the research method is different for the different characteristics of global anomalies and local anomalies ${ }^{[1]}$. Scholars have proposed some more classical methods to promote the continuous development of population anomaly detection technology.

For the global exception, it is necessary not only to detect whether the scene is abnormal, but also to judge the start and end of the abnormality and the intermediate transition phase. In general, the global anomaly detection method is to analyze the change of the event based on the motion estimation of the entire video picture.

Chen and Huang ${ }^{[2]}$ proposed a graph analysis algorithm based on eigenvalues. In their paper, each isolated area in the video picture is regarded as a vertex, and the whole group is regarded as a picture. Moreover, topological changes are simulated by local features (based on feature subgraph analysis and trigonometric transformation) and global features (time, etc.). Finally, the simulated topological variation characteristics are used to analyze the presence or absence of abnormal events in the population. 
Wu et al. ${ }^{[3]}$ proposed a population abscess detection method based on Bayesian model. The method is mainly based on the optical flow extraction motion feature, directly simulates the crowd movement with the conditional density function, and uses the Bayesian classification formula to determine whether there is an abnormal crowd escape event. Their experiments show that this method can accurately detect the abnormal behavior of the crowd, but it is not appropriate for the crowded scene. The global exception can only determine whether there is an abnormality in the monitoring screen. In actual applications, it is often necessary to locate the specific location where the abnormality occurs. Based on this, many scholars have proposed a local anomaly detection method, which usually divides the video picture into many small areas and locates the specific location of the abnormality through the abnormal situation of all small areas.

Biswas et al. ${ }^{[4]}$ proposed an abnormal event detection method based on the social force model. In the social force model, everyone in the group is simultaneously influenced by the individual's desired force and social interaction. The direction in which the individual expects the force indicates the direction of the movement desired by the individual, and the direction of the social interaction indicates the direction in which the environment, the pedestrian, etc. influence the individual. The direction of the two forces is the actual direction of the individual in the crowd. A particle flow calculation method based on optical flow is proposed to calculate the interaction force and solve the problem that the force calculation in the crowd is difficult due to serious crowding and occlusion. After modeling the crowd, the LDA model is used to determine the normal and abnormal frames in the video.

Cong et al. ${ }^{[5]}$ proposed anomalous event detection for the sparse reconstruction cost (SRC) model. In this method, three different types of multi-scale optical flow histograms are extracted for different local anomalous behaviors and global anomalous behaviors. After extracting features from normal frame images, the feature sets are composed of the extracted features, and the redundant information in the dictionary set is eliminated by an optimized method to form an optimal dictionary set. At the same time, the method uses the best dictionary set to judge whether each frame of the test set has abnormal behavior through the SRC method. Li et al. ${ }^{[6]}$ proposed a mixed dynamic texture model (MDT) to detect anomalies in dense populations. The MDT model performs a time-space block on a video sequence to detect whether an exception has occurred. In the time anomaly detection, the local distribution of the image intensity is simulated based on the foreground extraction of the Gaussian mixture model. In the spatial anomaly detection, the local region where an abnormality may occur is discriminated based on the principle of image saliency (the spatial position of the abnormality is higher than a certain threshold).

Song et al. ${ }^{[7]}$ proposed a chaotic invariant algorithm. This paper proposes a human flow model that is applicable in both structured and unstructured scenarios. First, the particles are advected based on the optical flow, and the trajectory of the human flow is represented by the trajectory of the aggregated particles. Then, all representative trajectories are quantified using a blunt invariant, and a model is trained using the quantized chaotic set. Finally, the maximum likelihood estimation is used to identify abnormalities and normal behaviors in the population.

Kratz and Nishino ${ }^{[8]}$ proposed a framework for modeling local temporal and spatial motion behaviors in dense population scenarios based on Hidden Markov Models (HMM). In the training phase, the temporal relationship between local motion patterns is extracted by a distribution-based HMM, and the spatial relationship is modeled by a coupled HMM. In the test phase, the anomaly event is the statistical deviation in the same scene in the video sequence. Their experiments show that HMM is suitable for analyzing more intensive scenes, and an HMM is established for each small area, which indicates that the method is only appropriate for a limited variety of normal behavior.

Despite the increasing popularity of video surveillance equipment and the maturity of intelligent surveillance technology, real-time problems are faced in practical applications. At present, although many 


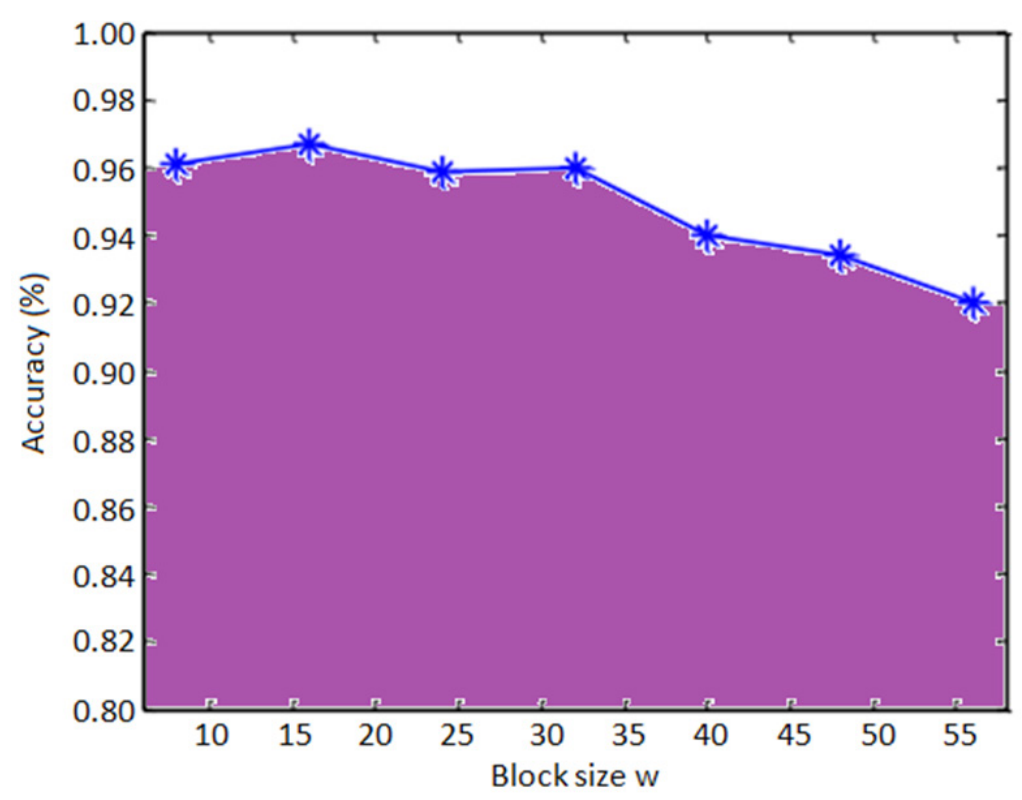

Figure 1. The algorithm's detection results are accurate under different block sizes ${ }^{[9]}$

algorithms achieve high accuracy on public datasets, it is difficult to meet real-time requirements due to, e.g., computational complexity. Thus, based on data mining, this study combined cloud computing image processing technology for real-time crime behavior recognition.

\section{METHODS}

\subsection{Population abnormal behavior detection}

To analyze the behavior of the crowd, each field is divided into a set of patches (blocks), and the population density in the image field determines the size of the block for the field. The purpose of this statistical partitioning of images by patches is mainly to better avoid interference from other factors. The proposed detection algorithm was tested on the public dataset UMN (University of Minnesota) to find the best block size. Figure 1 shows that, when the scale is higher than 40, the accuracy becomes larger with the block size, and the progress is basically stable. When the scale is higher than 40 , the accuracy begins to decrease. However, it should be noted that, if the block size is too large, the motion state cannot be described well. Therefore, the block size selected in this section is $24 \times 24$. The schematic is shown in Figure $2^{[10]}$.

This paper uses a foreground extraction algorithm based on K-means clustering. When the crowd escapes in the scene, the acceleration of the crowd changes. Therefore, this paper uses the magnitude of the acceleration to extract the foreground. When the amplitude of a position in the scene is greater than the threshold, the position is judged as the foreground. In this paper, the $\mathrm{K}$-means algorithm, with $\mathrm{k}=2$, is used to calculate the threshold value by randomly calculating the partial vector selected from the sample set $^{[11]}$.

Since the performance of abnormal behaviors is diverse, the definitions of exceptions are different in different scenarios. For any video image describing the behavior of the crowd, the sequence is described by the acceleration and velocity vectors. The basic steps of detecting the abnormal escape behavior of the crowd are as follows: (1) a given video image is smoothed by an image preprocessing method to remove noise; (2) the velocity and acceleration vectors are calculated using a modified acceleration algorithm for the processed grayscale image sequence. The improved acceleration algorithm combines the changes in the characteristics of the movement of the crowd and the changes in the distribution of the population. 


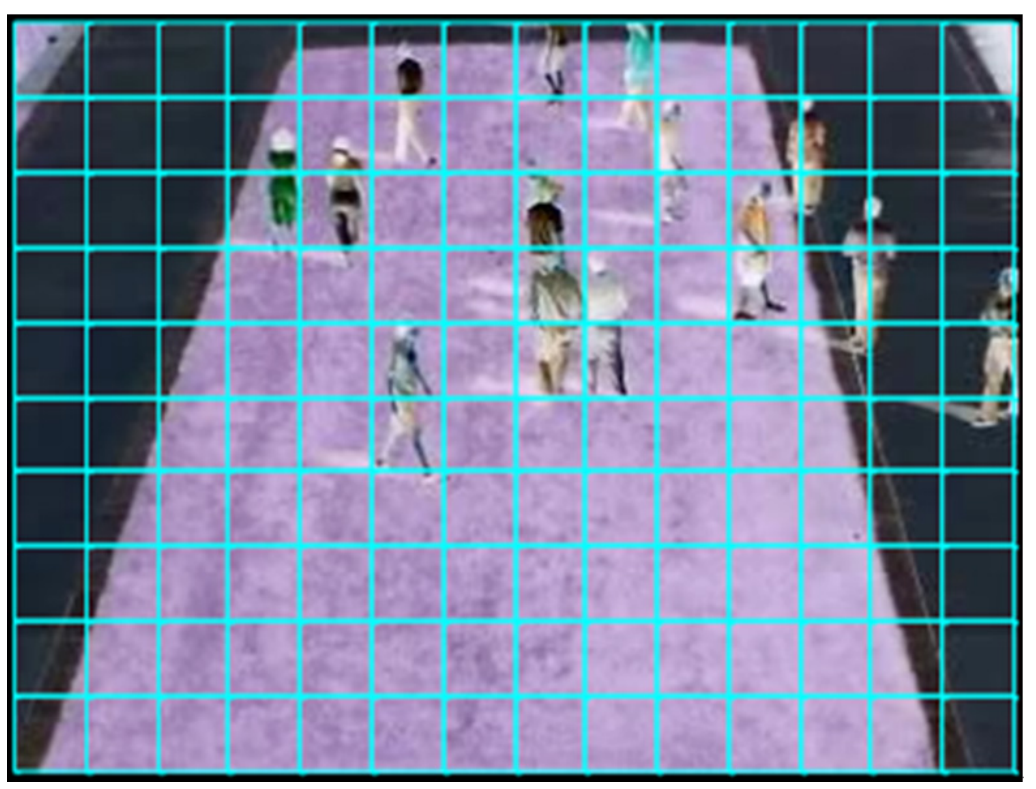

Figure 2. Schematic diagram of block processing of video frames

The neighborhood size for solving the acceleration in the experiment was $5 \times 5$; (3) regarding anomaly classification, most algorithms are based on empirical thresholds. The threshold $\varepsilon$ in this paper is mainly the continuous actual detection and analysis of different datasets; and (4) an optimal behavior determination based on an optimal threshold is applied to a given video sequence. If it is greater than the threshold, it is considered that there is an abnormal situation; otherwise, it is considered a normal video frame. Represent threshold as Ai. The detection process can be described as ${ }^{[12]}$ :

$$
F\left(A_{i}\right)=\left\{\begin{array}{c}
\text { normal }, A_{i}<\varepsilon \\
\text { anomaly, } A_{i} \geq \varepsilon
\end{array}\right.
$$

In real video images, the crowd leisurely walks in the direction they want to go. However, when there are unexpected events, people will run around because of psychological panic. At this point, people usually get away from danger quickly. This results in a very significant change in the speed of the human body, thus the range of motion of the moving object is also large.

It should be noted that both normal behavior and abnormal behavior are continuous; they cannot occur at a certain moment, and then disappear immediately. Therefore, occasional short-lived anomalous frames that appear in normal video frames are still considered normal video frames. Conversely, several frames of normal frames that occur by chance in an abnormal video frame are still determined as abnormal video frames ${ }^{[13]}$.

\subsection{Escape center}

The anomaly detection object in this paper is a random group in ordinary public places. That is to say, the movement direction of the crowd is irregular when there is no abnormality. Usually, sudden abnormal situations for ordinary people will bring fear to people and then move away from the point where the anomaly occurs. Therefore, according to this principle, the idea of escaping from the center is introduced, and the place where the abnormality may occur is determined by determining the escape center. As shown in Figure 3, one or more anomalies may occur in a scene. The squares in the figure represent moving targets, the arrows on the squares indicate the direction of motion of the moving targets, and the blue dots represent the locations where anomalies may occur ${ }^{[14]}$. 

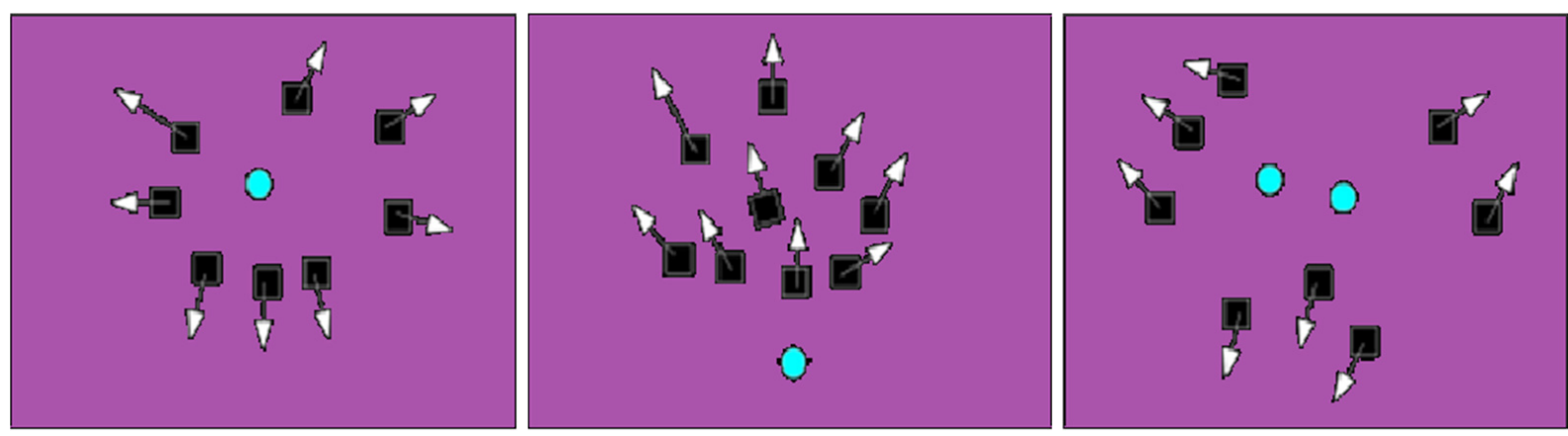

Figure 3. Schematic diagram of the escape center

\subsection{Anomaly localization algorithm based on single escape center}

According to the introduction to the escape center in the previous section, the escape center can be obtained from the movement information of the crowd in the specific case studied in this paper. The population studied in this paper is mainly people who have fled after being affected by emergencies in public places. Moreover, anomaly detection and localization for this situation can be implemented by combining the acceleration features with the escape center. First, the acceleration feature extraction is performed through the optical flow field of the video image to determine whether an abnormality has occurred. If there is a possibility of an abnormality, the escape center position is calculated. Moreover, it is determined that the center position can be escaped by using the intersection of all the acceleration vector inverse extension lines obtained by detecting the position and direction information of the acceleration vector when the abnormality is detected and using $\mathrm{K}$ nearest neighbor search. Simulation experiments show that the method can well determine the possible location of anomalies in simple places where anomalies occur ${ }^{[15]}$.

The anomaly localization algorithm in this paper is based on the analysis of the motion vector of the moving target in the abnormality detection process to locate the position where the abnormality may occur. The process of locating mainly consists of counting all the intersections of the inverse extension lines of the motion vector and using the $\mathrm{K}$ nearest neighbor search method to determine the most likely abnormal $\operatorname{position}^{[16]}$.

The algorithm is as follows: (1) by framing the video, the image sequence is obtained, and then the motion feature vector is extracted; (2) each frame of the image is subjected to improved acceleration signature calculations, and an improved acceleration threshold is obtained through a number of experiments. The acceleration vector of this frame image is retained if the improved acceleration value is greater than the threshold; (3) according to the obtained acceleration vector $\mathrm{A}\left(\mathrm{a}_{\mathrm{x}}, \mathrm{a}_{\mathrm{y}}\right)$ of the image, the corresponding onedimensional linear equation parameter is calculated. When ignoring special cases, the parameter expression is as follows ${ }^{[17]}$ :

$$
\begin{aligned}
& k_{l}=a_{y(i, j)} / a_{x(i, j)} \\
& b_{l}=i-k_{l} \times j
\end{aligned}
$$

where $i$ and $j$ represent the positions of the corresponding pixels in the image; (4) According to the linear equation of the acceleration vector, the intersection point $P=\left\{p_{1}, p_{2}, \ldots p_{s}\right\}$ is calculated. The main calculation is the intersection of all the two straight lines, and the mathematical derivation is as follows ${ }^{[18]}$ :

$$
\begin{aligned}
& x=\left(b_{2}-b_{1}\right) /\left(k_{1}-k_{2}\right) \\
& y=\left(\left(b_{2}-b_{1}\right) /\left(k_{1}-k_{2}\right)\right) \times k_{1}+b_{1}
\end{aligned}
$$

(5) there are many repetitions in the process of calculating the intersection set. The intersections that do not match the actual situation are collectively referred to as wild intersections. Therefore, to improve the 


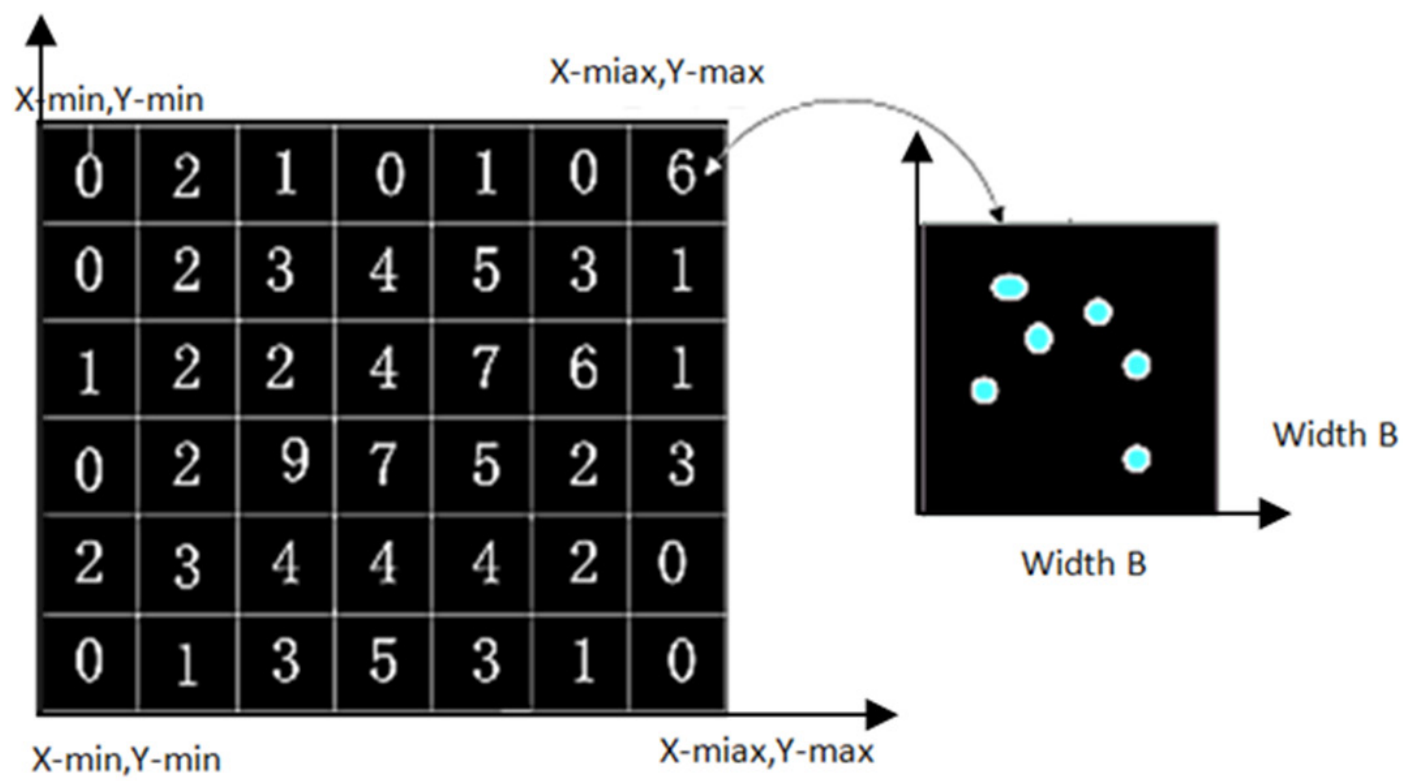

Figure 4. Block diagram of the principle of graphic method

accuracy of the algorithm, it is necessary to remove wild intersection points from the intersection point set; and (6) by further analyzing the intersection set, the escape center is determined. Since the intersection set referred to in this paper belongs to a simple dataset, the K nearest point search method can be used.

The $\mathrm{K}$ nearest point search method is also known as the $\mathrm{K}$ nearest neighbor search method. In the process of determining the escape center, this search method calculates the distance between the intersections in the neighborhood, selects the Kth minimum distance in the distance set of each intersection, and compares it with other intersections to determine whether it is the escape center. This search method is suitable for the classification of rare events. Since the escape center in this section is an infrequent event, and this search method is easy to implement without training, this paper uses the K nearest neighbor search method to determine the escape center of abnormal populations ${ }^{[19]}$.

In determining the escape center, the determination of the intersection of the lines of the improved acceleration vector in the image plays a very important role in the overall algorithm. In the process of determining the intersection, all intersections are calculated and the wild intersections are removed. The calculation method of the intersection point is introduced above, and the removal process of the intersection point is introduced here.

The wild intersection removal method in this paper adopts the graphical removal method, as shown in Figure 4. The specific steps of the algorithm are as follows ${ }^{[20]}$ : (1) the intersection set $P=\left\{\mathrm{p}_{1}, \mathrm{p}_{2}, \ldots \mathrm{p}_{s}\right\}$ solved by the improved acceleration vector is taken as an input; (2) the overall scope of the graphical search is determined; and (3) a small search window is designed to count the number of intersections in the search range determined in the previous step. The size of the small search window is determined before the start of the detection and is mainly determined according to the number of moving objects in the image and the size of the image.

The graphic method removes the wild intersection points mainly based on the number of intersection points in the search window to make the intersection point, which is mainly determined by the particularity of the research object of the algorithm. Moreover, the algorithm is mainly used to locate the 
A

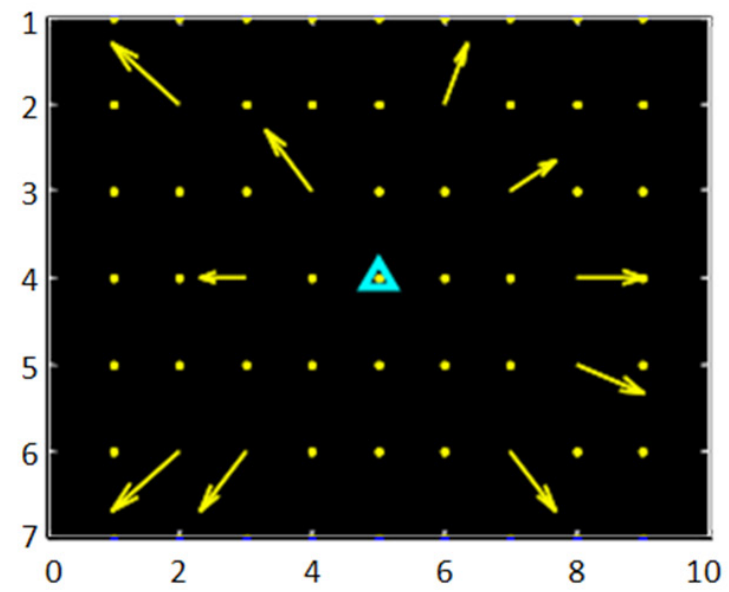

B

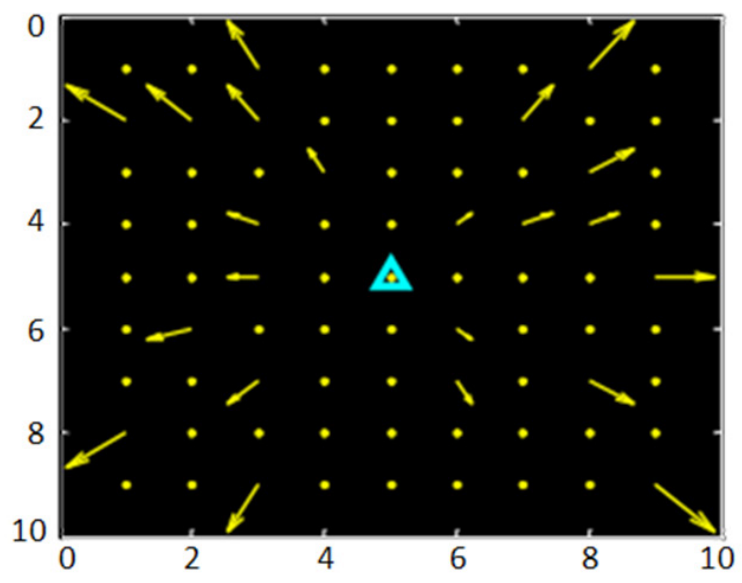

Figure 5. Escape center obtained in the simulation data experiment. A: center test result 1; B: center test result 2

A

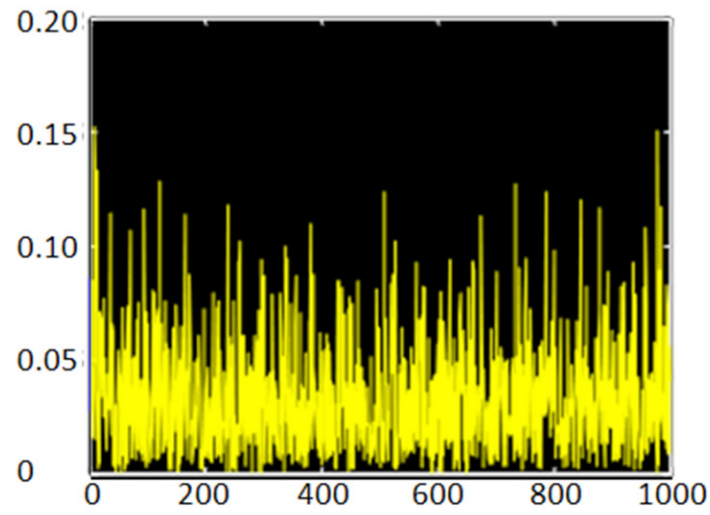

B

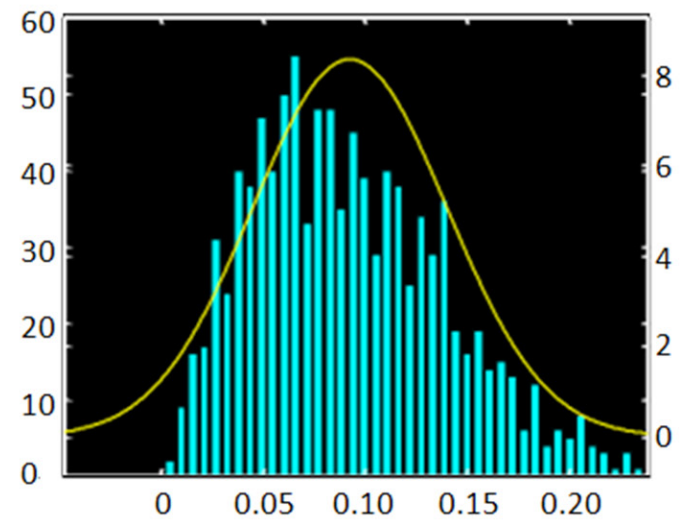

Figure 6. Curve image of deviation. A: variation of deviation; B: Histogram and Gaussian fitting curve of deviation

escape center of the crowd, and the escape center is defined as the position where the intersection of the motion vector is the densest. Therefore, the removal of wild intersections should remove a smaller number of intersections in the search window. The graphical method used in this paper is obtained through many experiments, and the empirical threshold is used to measure the intensity of intersections to assist the removal of wild intersections.

The limitations of individual motion in the population detected herein are often referred to as directional noise, and such noise is subject to a Gaussian distribution. Therefore, according to the actual situation of the research object in this paper, a set of random numbers with five-degree direction noise is designed to be added to the synthesized data, so that the synthesized data are a set of known ideal data of the escape center close to the actual situation. The test results are shown in Figure 5.

The accuracy of the method is further tested by the deviation of the statistical positioning results, as shown in Figure 5.

In Figure $6 \mathrm{~A}$, the abscissa indicates the number of experiments, and the ordinate indicates the mean square error. By calculating the average deviation of the 1000 experiments, the average deviation of the proposed 
algorithm in the experimental data of adding direction noise is 0.0292 . This shows that the algorithm has high positioning accuracy and strong anti-interference ability. It can be seen in Figure $6 \mathrm{~B}$ that the deviation generally obeys a right-skewed distribution. Through the above simulation experiments, it can be seen that the abnormal positioning of a single escape center is highly accurate in the ideal synthetic data experiment.

The optical flow field of the video image is used to perform the acceleration feature extraction to determine whether an abnormality occurs. If there is an abnormality, there is escape from the center location. The position and direction information of the acceleration vector obtained when the abnormality is detected is used to obtain all the acceleration vector reverse extension lines Intersection, and then $\mathrm{K}$ nearest neighbor search is used to determine the possible escape from the center position.

The anomaly localization algorithm in this paper is based on the analysis of the motion vector of the moving target in the anomaly detection process to locate where the anomaly may occur. The process of locating is mainly by counting all the intersections of the inverse extension lines of the motion vector, and using the $\mathrm{K}$ nearest neighbor search method to determine the most likely abnormal location. The algorithm is as follows:

(1) Obtain the image sequence by framing the video, and then extract the motion feature vector.

$$
\begin{aligned}
& A_{X}^{n}=\left\{a_{x 1}^{(n)}, a_{x 1}^{(n)}, \ldots, a_{x k}^{(n)}\right\} \\
& A_{Y}^{n}=\left\{a_{y 1}^{(n)}, a_{y 1}^{(n)}, \ldots, a_{y k}^{(n)}\right\}
\end{aligned}
$$

(2) Calculate the improved acceleration characteristics for each frame of image, and obtain the improved acceleration threshold through many experiments. If the improved acceleration value is greater than the threshold, the acceleration vector of this frame of image is retained.

(3) Calculate the corresponding univariate linear equation parameters according to the acceleration vector $\mathrm{A}\left(\mathrm{a}_{\mathrm{x}}, \mathrm{a}_{\mathrm{y}}\right)$ of the obtained image; ignoring the special case, the parameter expression is as follows:

$$
\begin{gathered}
k_{l}=a_{y(\mathrm{i}, \mathrm{j})} / a_{x(\mathrm{i}, \mathrm{j})} \\
b_{l}=i-k_{l} \times j
\end{gathered}
$$

where $i$ and $j$ represent the positions of corresponding pixels in the image.

(4) Calculate the intersection point set $P=\left\{p_{1}, p_{2}, \ldots p_{s}\right\}$ according to the straight-line equation of the acceleration vector. In order for the intersection point set to include all intersection points, the intersection points of all two different straight lines are mainly calculated. The mathematical derivation is as follows:

$$
\begin{gathered}
x=\left(b_{2}-b_{1}\right) /\left(k_{2}-k_{1}\right) \\
y=\left(b_{2}-b_{1}\right) /\left(k_{2}-k_{1}\right) \times k_{l}+b_{1}
\end{gathered}
$$

(5) In the process of calculating the intersection set, there are many repetitions or intersections that are inconsistent with the actual situation, which are collectively called wild intersections. Therefore, to improve the accuracy of the algorithm, wild intersections need to be removed from the intersection set.

(6) Determine the escape center by further analyzing the intersection set. Since the intersection set involved in this article belongs to a simple dataset, the $\mathrm{K}$ nearest point search method can be used. The $\mathrm{K}$ nearest point search method is also known as the $\mathrm{K}$ nearest neighbor search method. In the process of determining the escape center, this search method is to calculate the distance between intersections in the neighborhood, select the Kth smallest distance in the distance set of each intersection, and then compare them with other intersections to determine if they escaped the center. This search method is suitable for the classification of rare events. Since the escape center in this section is an infrequent event, and this search method is easy to implement without training, this paper uses the $\mathrm{K}$ nearest neighbor search method to determine the escape center of the abnormal crowd.

The graphical method of removing wild intersections is mainly based on the number of intersections in the search window. Intersections are rounded off, which is mainly determined by the particularity of the 


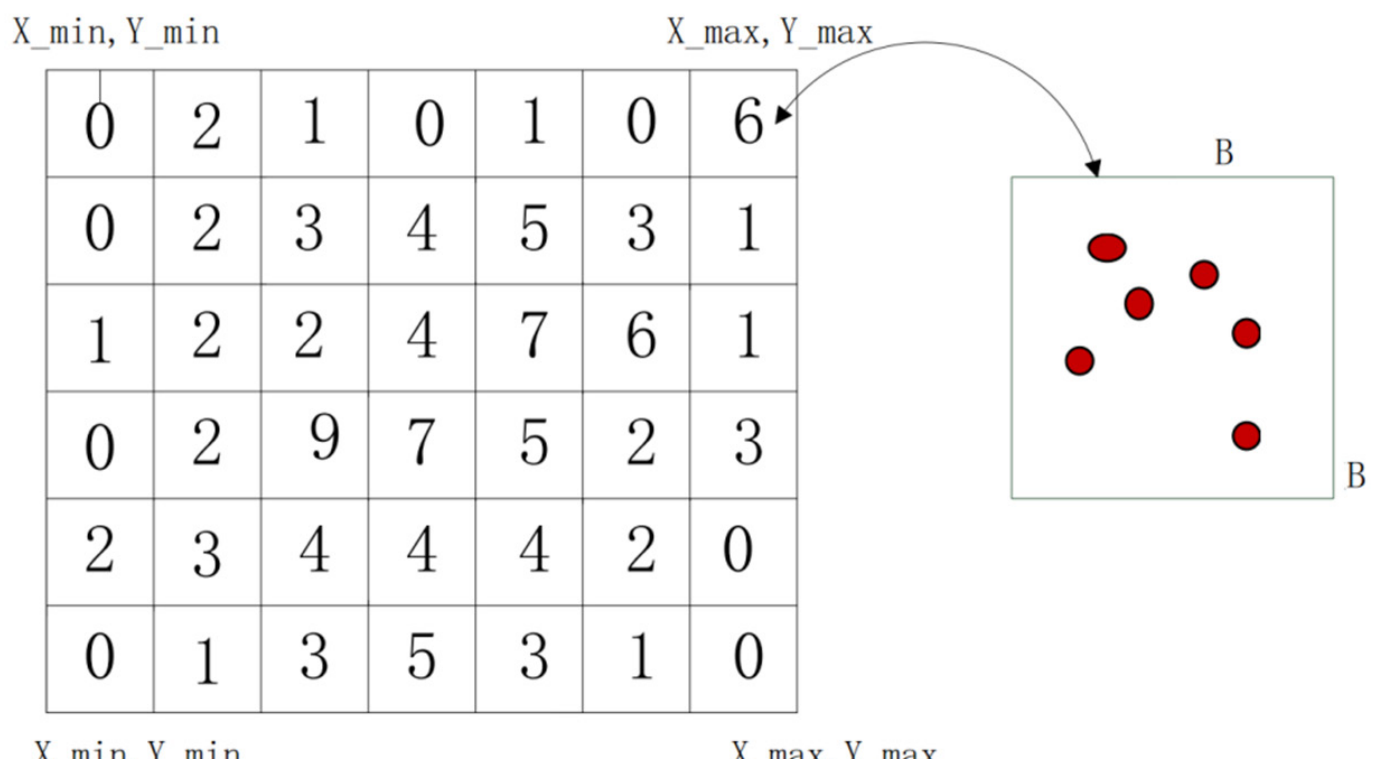

$\mathrm{X} \min , \mathrm{Y} \min$

$\mathrm{X} \max , \mathrm{Y} \max$

Figure 7. Schematic block diagram

research object of this algorithm. The algorithm is mainly used to locate the escape center of the crowd, and the escape center is defined as the position where the intersection of the motion vector is the densest. Therefore, the removal of wild intersections should remove a smaller number of intersections in the search window. The graphical method used in this paper uses many experiments to obtain empirical thresholds to measure the denseness of intersections to assist in removing wild intersections [Figure 7].

\section{EXPERIMENTAL EVALUATION}

Step 1: Select the video. In the experiments, 10 segments of video were selected to test three kinds of abnormal behaviors. The test video mainly came from a bank monitoring video, and some videos were from the crime scene monitoring. Moreover, the surveillance video was recorded by Haikang or Dahua cameras. Some test videos were taken by digital cameras, and these video formats were different, including AVl, MP4, DAV, etc. In addition, the system unified the video sources to AVI format for easy processing. The number of pedestrians appearing in the selected video, the time of appearance of the pedestrian, the video resolution, etc. were all random and there was no law. In addition, because shadow removal is not the focus of this paper, to reduce the impact of shadow on the algorithm, only video with less obvious shadow was selected when selecting video.

Step 2: Parameter configuration. First, the camera corresponding to the selected video was calibrated, and after the coordinate conversion was completed, the actual coordinate matrix was saved as an XML file (the file name is the camera name, and the file suffix is .xml). Then, the estimated target imaging size, frame interval, monitoring area, abnormal behavior type, etc. were saved in the PAR file (the file has the same name as the test video file and the file suffix is .par).

Step 3: The result of manual statistics. The video was observed by the human eye, and the manual statistical result was recorded in the MTR file (the file has the same name as the test video file, and the file suffix is .mtr). Manual statistics were needed to record the type of abnormal behavior, start time, and end time. Finally, the number of samples with abnormal behavior (abnormality) and no abnormal behavior (normal) in the selected video were counted according to the behavior type. The statistical results are shown in Tables 1-3. 
Table 1. Number of manual statistical samples of regional invasive behavior

\begin{tabular}{|c|c|c|c|c|c|c|c|c|c|c|c|}
\hline & V1 & V2 & V3 & V4 & V5 & V6 & V7 & V8 & V9 & V10 & Total \\
\hline Abnormal & 14 & 8 & 26 & 12 & 17 & 8 & 8 & 5 & 18 & 8 & 124 \\
\hline Normal & 3 & 8 & 0 & 5 & 5 & 3 & 3 & 0 & 3 & 3 & 33 \\
\hline
\end{tabular}

Table 2. Number of manual statistical samples of trailing behavior

\begin{tabular}{|c|c|c|c|c|c|c|c|c|c|c|c|}
\hline & F1 & F2 & F3 & F4 & F5 & F6 & F7 & F8 & F9 & F10 & Total \\
\hline Abnormal & 6 & 8 & 14 & 11 & 8 & 3 & 2 & 3 & 8 & 5 & 68 \\
\hline Normal & 3 & 5 & 5 & 6 & 2 & 3 & 0 & 3 & 0 & 3 & 30 \\
\hline
\end{tabular}

Table 3. Number of manual statistical samples of defamation behavior

\begin{tabular}{|c|c|c|c|c|c|c|c|c|c|c|c|}
\hline & T1 & T2 & T3 & T4 & T5 & T6 & T7 & T8 & T9 & T10 & Total \\
\hline Abnormal & 8 & 6 & 11 & 3 & 2 & 5 & 8 & 9 & 6 & 12 & 70 \\
\hline Normal & 6 & 2 & 5 & 0 & 2 & 3 & 5 & 5 & 3 & 2 & 33 \\
\hline
\end{tabular}
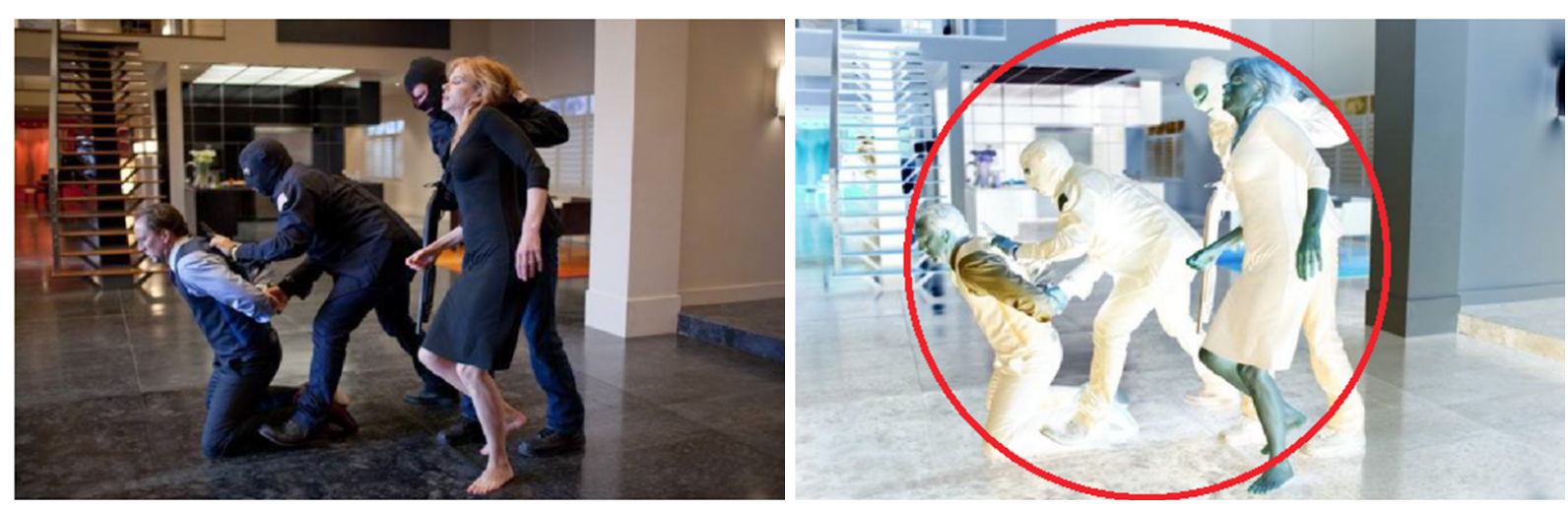

Figure 8. Identification of illegal intrusion crimes

In the detection of regional intrusion behavior, the algorithm does not use actual coordinates, but directly judges according to pixel coordinates. Therefore, there is no difference between the algorithm and the traditional algorithm in the abnormal behavior analysis stage. However, because the algorithm in this paper is better than the traditional algorithm in the target detection and target tracking stage, the false negative rate of the algorithm is greatly reduced, and finally higher accuracy is achieved. For the analysis of the video with false positives, it is easy to have false positives in the following cases: Pedestrians walk outside the edge of the surveillance area, and the feet do not enter the surveillance area but are closer to the boundary of the surveillance area, as shown in Figure 8.

One key to detecting trailing behavior is the setting of the relative distance and relative distance threshold between two pedestrians. The traditional algorithm is processed based on the pixel coordinate trajectory, thus the relative distance and the distance threshold can only be calculated by the number of pixel points, which is only an estimated value and is not accurate. However, the proposed algorithm is based on the actual coordinate trajectory, and the actual distance between the targets can be calculated, which is used as the distance threshold. In the test, the distance threshold of the algorithm was $T_{d}=1000 \mathrm{~cm}$, and the distance threshold of the traditional algorithm was $T_{d}=100$ pixels, as shown in Figure 9.

Awkward behavior is a relatively complex behavior that requires not only the calculation of the speed of pedestrian movement, but also the calculation of the angle of pedestrian movement. While the traditional algorithm is based on the pixel coordinate trajectory, the proposed algorithm is based on the actual coordinate trajectory [Figure 10]. 

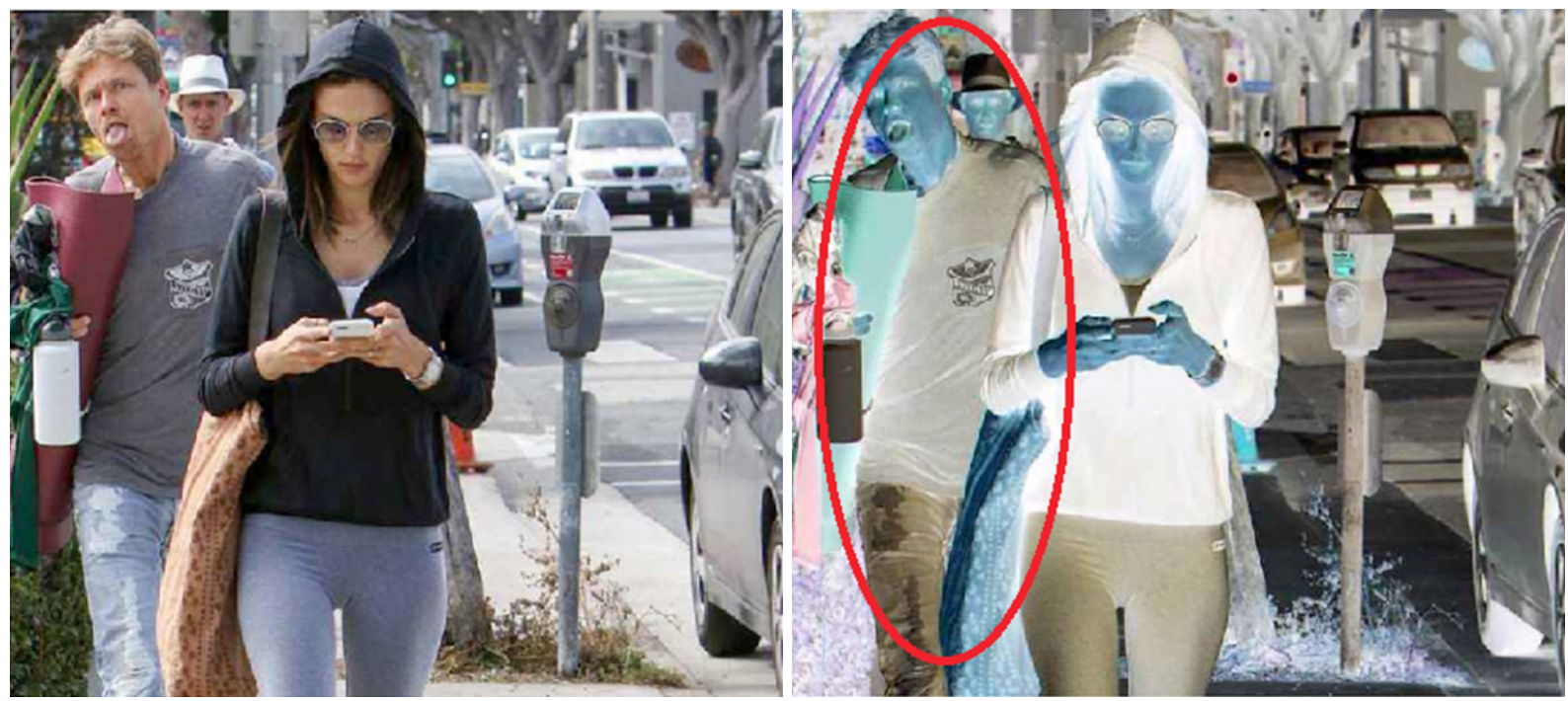

Figure 9. Trailing criminal behavior identification
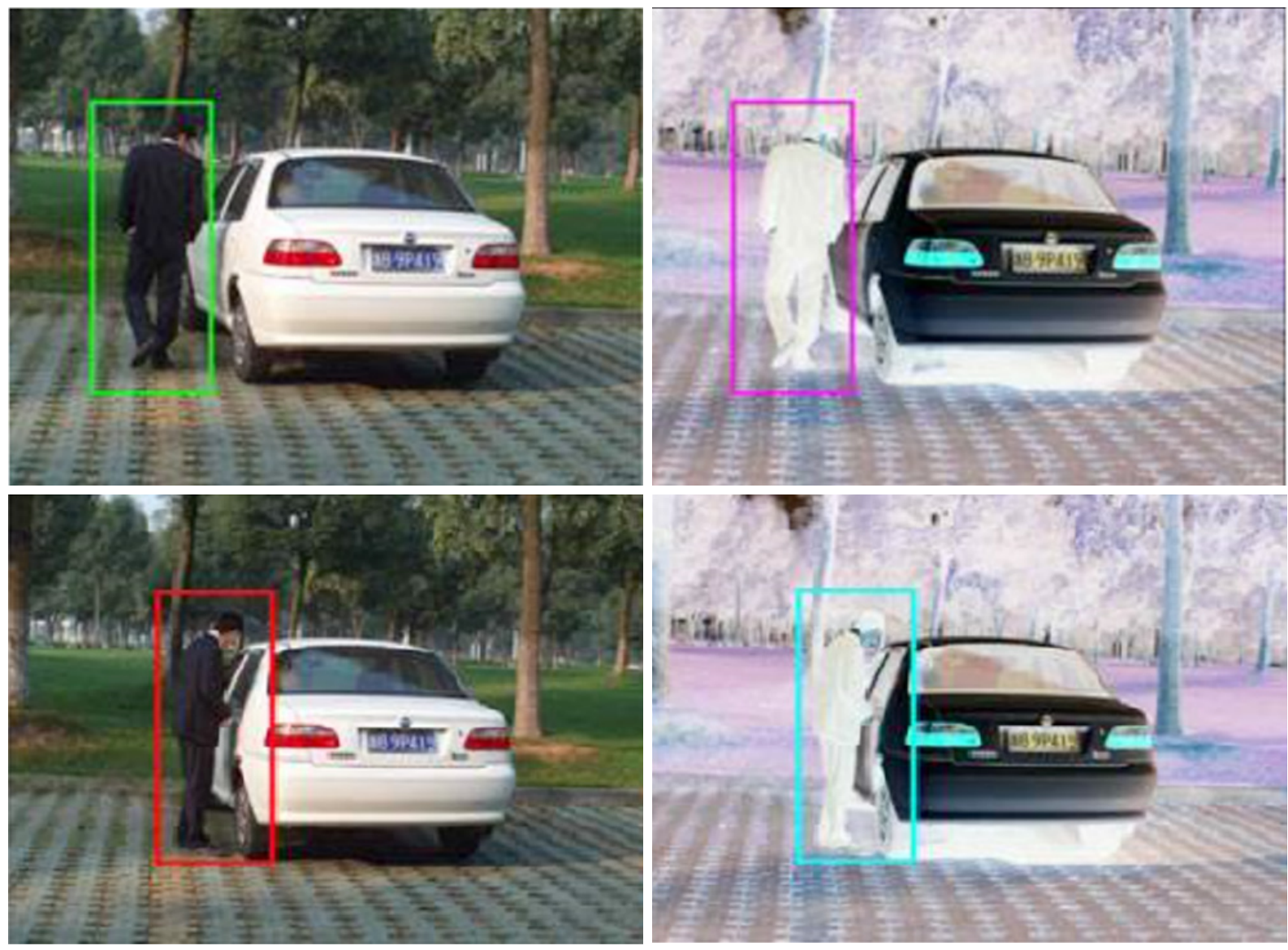

Figure 10. Identification of wandering criminal behavior

To further verify the performance of the algorithm, the UMN video library and PETS200 video library were used to perform simulation experiments on algorithm performance verification. First, the feasibility of the algorithm was verified at the theoretical level by using the set ideal dataset for simulation experiments. 

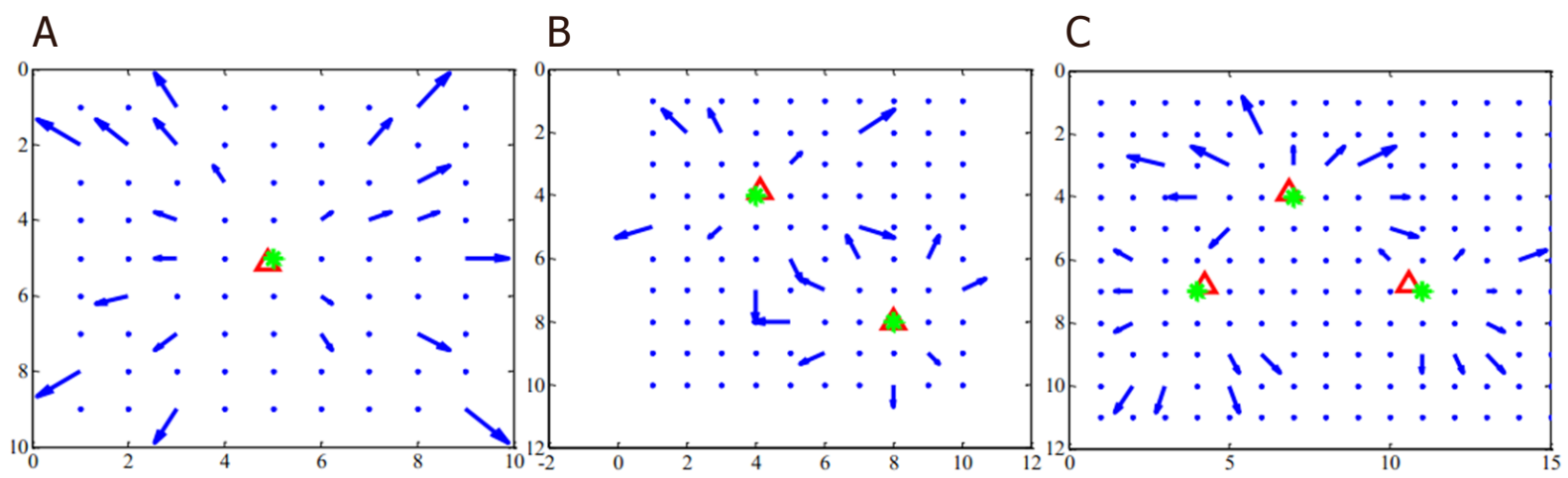

Figure 11. Results of escaping from the center position in the synthesized vector field. A: one escape center; B: two escape centers; C: three escape centers

As shown in Figure 11, the algorithm was used to perform different positioning experiments on different numbers of escape centers. Considering that it is difficult to have more than three sudden escape events in actual scenes, the research situation was a simple incident.

Since the data used in the simulation experiment were specifically designed, the red mark and green mark in Figure 11, respectively, represent the detected escape center and the ideally preset escape center. The detection of ideal data can verify that the algorithm is theoretically feasible. To make the designed data closer to an actual dataset, the same method as the previous simulation experiment to add Gaussian direction noise was chosen. It was found through experiments that the addition of directional noise did not affect the accuracy of the algorithm much. However, from the above simulation experiments, it can be seen that, for the three scenarios of escaping the center, the selection of K value by the KNN search method during the process of escaping the center and removing the wild intersections has a certain impact on the performance of the entire algorithm. These two factors that may affect the performance of the algorithm were analyzed further, as shown in Figures 12-14.

As shown in Figure 12, the overall positioning performance of the algorithm is still very high. Figures 13 and 14 show the change of the mean square error of the positioning error caused by the selection of different $\mathrm{K}$ values during the algorithm's search process for escaping the center and the process of removing wild points. It can be seen from the fitting curve in Figure 13 that the accuracy of selecting an appropriate $\mathrm{K}$-value algorithm corresponding to different directional noise angles can be higher. Figure 14 is a comparison diagram between the searching wild intersection removal method and the graphical method. It can be seen in the figure that properly selecting the value of $\mathrm{K}$ under a fixed noise angle can make the performance of the algorithm in this section better.

In order to test the effect of this research algorithm in the actual crime detection, through the intelligent identification of 60 sets of crime surveillance videos, the identification effect of crime actions is counted. The crime recognition accuracy rate is shown in Table 4 and Figure 15.

As shown in Figure 15, this algorithm has a high recognition rate for criminal actions, and has certain practical significance. It can be applied to practice.

\section{DISCUSSION}

This study verified the accuracy of the abnormal positioning method of the single escape center through simulation experiments. First, this study verified the accuracy of positioning under synthetic data, which mainly proved the feasibility of the theory. Then, this study validated the positioning method using 


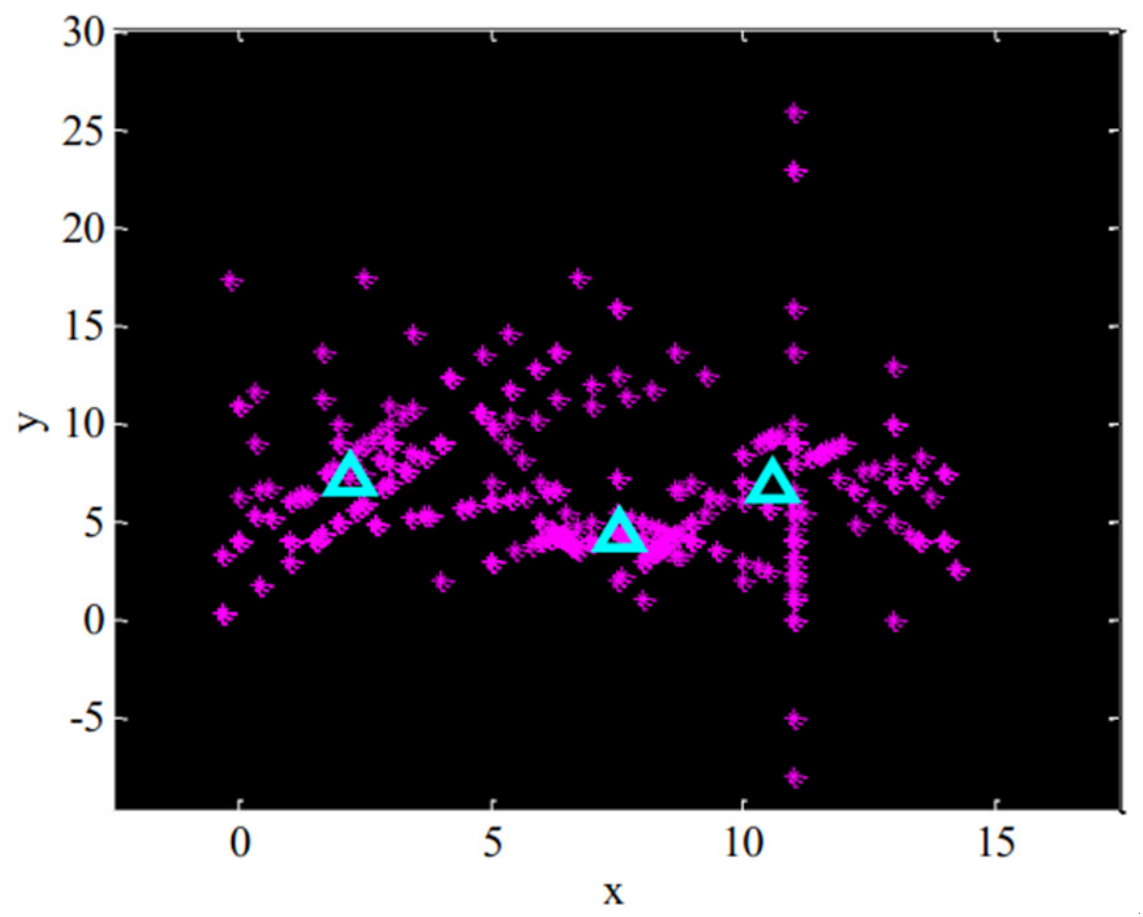

Figure 12. Location of the simulated escape center in the intersection diagram

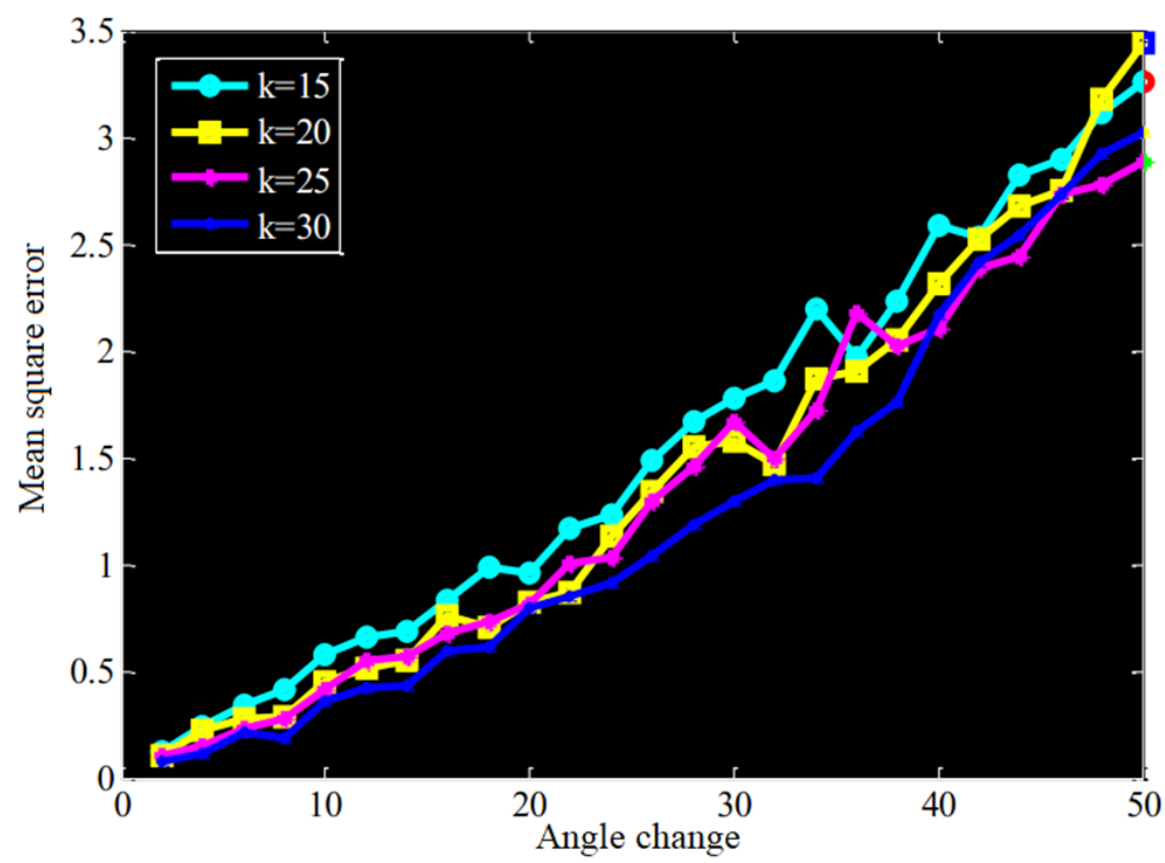

Figure 13. Influence of $K$ value on detection results

the UMN dataset. This experiment was mainly to verify the accuracy of the algorithm in the actual environment.

The concept of "escape center" is used to approximate the abnormal scattered behavior of the crowd. According to the state of the crowd distribution index and acceleration characteristics on the playing field, 


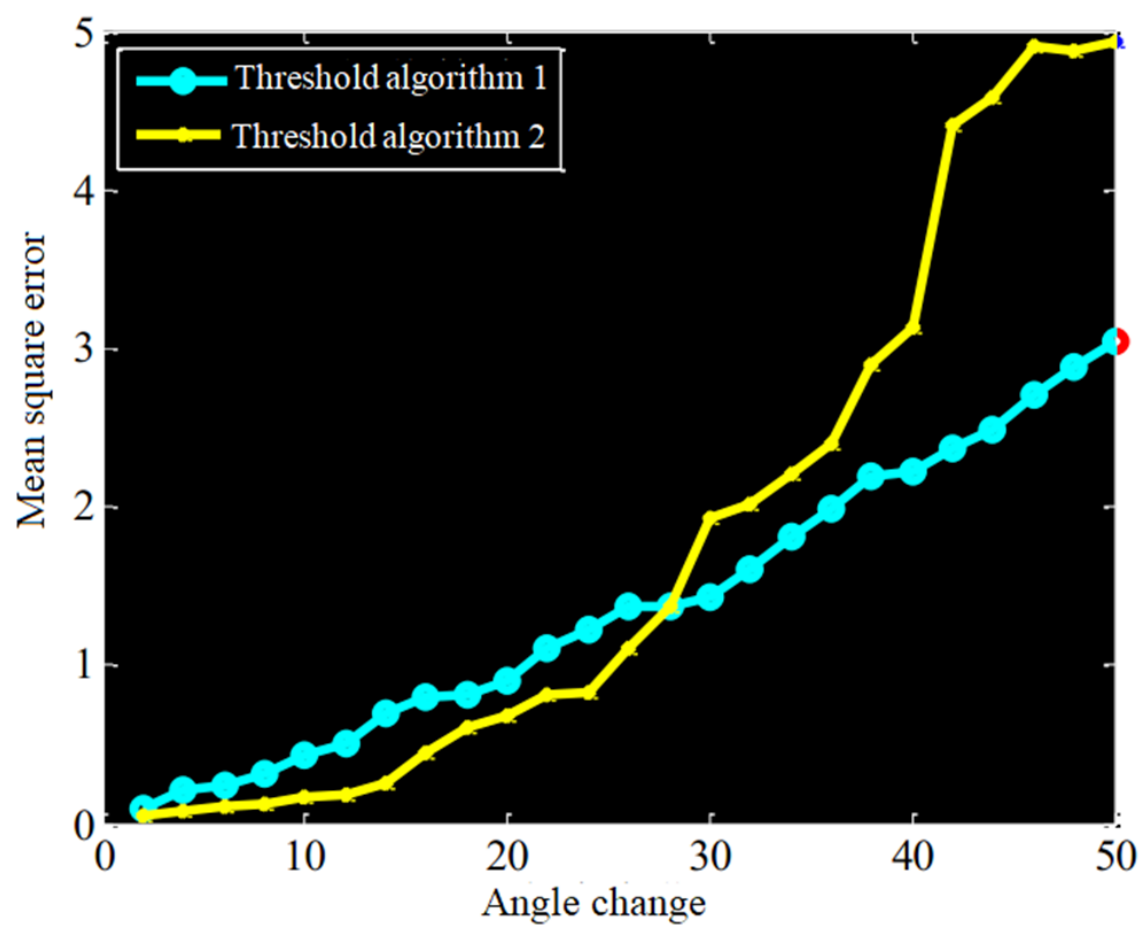

Figure 14. Comparison of errors of different field removal methods

Table 4. Statistical table of crime identification accuracy (\%)

\begin{tabular}{|c|c|c|c|c|c|}
\hline No. & $\begin{array}{c}\text { Crime identification accuracy } \\
\text { rate (\%) }\end{array}$ & No. & $\begin{array}{c}\text { Crime identification accuracy } \\
\text { rate (\%) }\end{array}$ & No. & $\begin{array}{c}\text { Crime identification accuracy } \\
\text { rate (\%) }\end{array}$ \\
\hline 1 & 84 & 21 & 92 & 41 & 85 \\
\hline 2 & 92 & 22 & 89 & 42 & 89 \\
\hline 3 & 84 & 23 & 91 & 43 & 87 \\
\hline 4 & 84 & 24 & 84 & 44 & 94 \\
\hline 5 & 82 & 25 & 90 & 45 & 93 \\
\hline 6 & 86 & 26 & 83 & 46 & 80 \\
\hline 7 & 91 & 27 & 92 & 47 & 82 \\
\hline 8 & 82 & 28 & 85 & 48 & 86 \\
\hline 9 & 87 & 29 & 82 & 49 & 91 \\
\hline 10 & 82 & 30 & 85 & 50 & 95 \\
\hline 11 & 82 & 31 & 92 & 51 & 92 \\
\hline 12 & 88 & 32 & 93 & 52 & 94 \\
\hline 13 & 87 & 33 & 88 & 53 & 93 \\
\hline 14 & 83 & 34 & 86 & 54 & 85 \\
\hline 15 & 92 & 35 & 84 & 55 & 93 \\
\hline 16 & 90 & 36 & 89 & 56 & 90 \\
\hline 17 & 94 & 37 & 90 & 57 & 90 \\
\hline 18 & 85 & 38 & 88 & 58 & 95 \\
\hline 19 & 92 & 39 & 87 & 59 & 83 \\
\hline 20 & 82 & 40 & 88 & 60 & 83 \\
\hline
\end{tabular}

the occurrence of anomalies was detected, and the location algorithm of the escape center was studied. Firstly, the algorithm to detect the single escape center was implemented. To further detect the possible location of anomalies in the actual scene, based on the single escape center, further research was conducted to obtain multiple localization algorithms for the escape center. Many experiments were performed on the synthetic data and the UMN public dataset. The experiments show that the algorithm based on the multiescape center is accurate in different scenarios. 


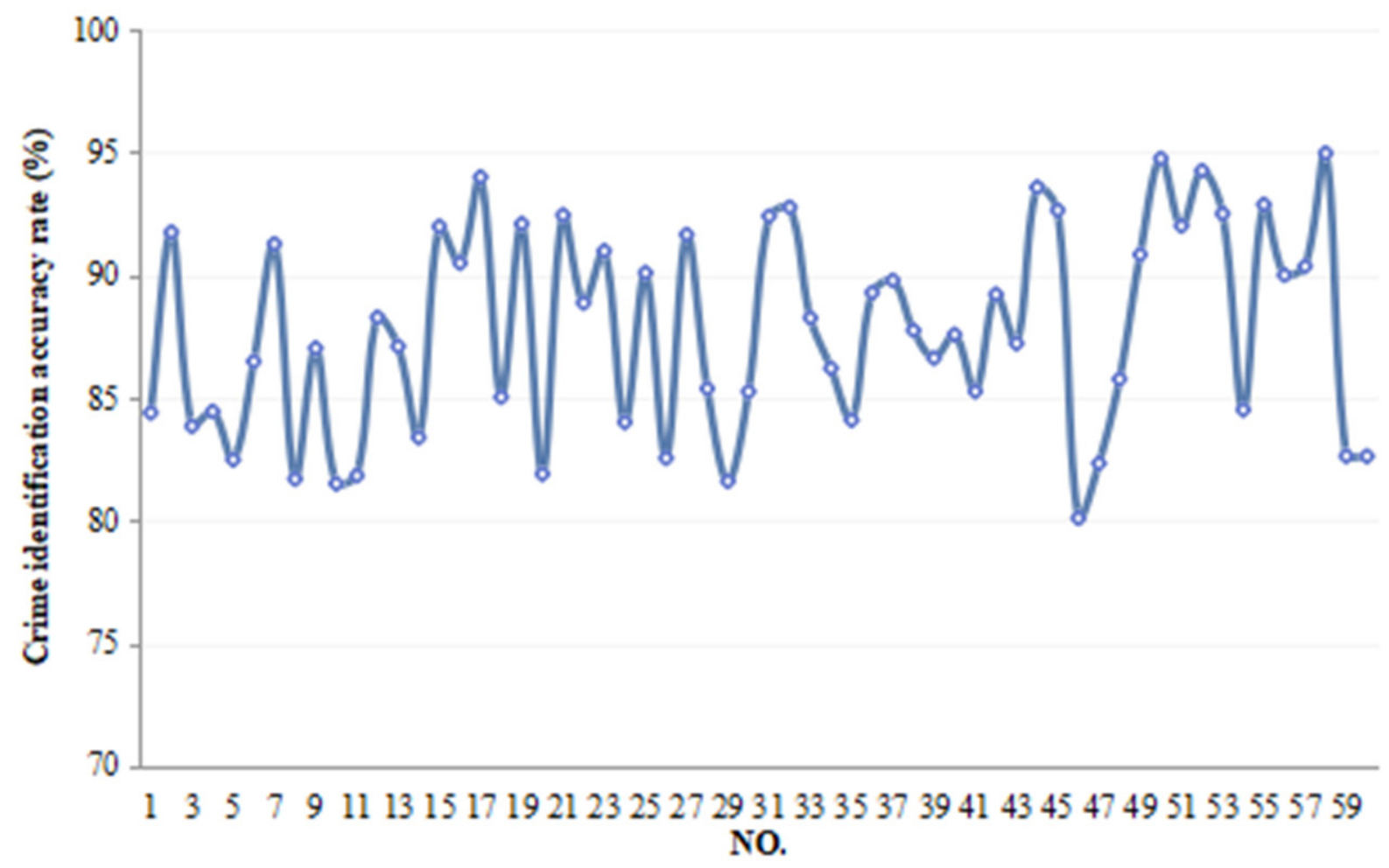

Figure 15. Statistics of crime recognition accuracy rate

In the actual public places, everyone will be affected by the movements of people around them and adjust their movement direction. The restriction of this group of people on individual movements must exist in a certain number of places. Therefore, the synthetic data used in the verification of the anomaly location method of the escape center should include the interference of the crowd limiting factor.

In view of the accuracy and real-time requirements of crime behavior identification, this study tested the accuracy and time consumption of the algorithm. Accuracy test calculated the system's false negative rate, false positive rate, and accuracy by comparing system analysis results with manual statistical results. The algorithm time consumption was evaluated by averaging the processing time per frame. The accuracy test process mainly included steps of selecting video, parameter configuration, manual statistical results, pixelbased traditional algorithm testing, algorithm testing, and comparative analysis.

As shown in Figure 7, when the algorithm detects the intrusion behavior of the area, it judges whether the left and right places of the pedestrian's external rectangular frame are in the monitoring area. If there is a certain location in the monitoring area, it is considered that there is a regional intrusion. Although the left and right positions of the rectangle are very close to the position of the two feet of the person, the position of the foot cannot be completely replaced correctly, which is also the reason for the false positive of the behavior. Although there are certain false positives in this method, the overall accuracy is high, and the method is simple and convenient. Therefore, it can meet the accuracy requirements of the security system.

It can be concluded from the results in Figure 8 that the accuracy of the proposed algorithm $\mathrm{i}$ is significantly improved compared with the traditional algorithm. The number of pixels corresponding to the actual distance of $1 \mathrm{~m}$ is affected by factors such as the angle at which the camera is mounted and the distance from the camera. Moreover, a uniform estimate is difficult to apply to any area of an image. When a pedestrian moves relative to the camera, the number of pixels separated by the two pedestrians' changes. 
Therefore, when using traditional algorithms, it is easy to have false positives or false negatives. The algorithm uses the actual distance threshold and judges the distance between the actual trajectory points of the two pedestrians, which is not affected by any factors, thus the accuracy is higher.

It can be concluded from the results in Figure 10 that the accuracy of the proposed algorithm is significantly improved compared with the traditional algorithm. The traditional algorithm calculates the movement speed and movement angle of pedestrians based on the pixel coordinate trajectory. However, since the camera in the security scene is generally fixed on a vertical wall rather than directly above the surveillance scene, the angles of the pixels in the video tend to be different from the true angles. In addition, the threshold in the traditional algorithm is set by the number of pixels according to experience, which has no practical significance, thus the accuracy of the traditional algorithm is not high. In summary, the algorithm has high accuracy in identifying abnormal behaviors and has high practical value, which can meet the accuracy requirements of security systems.

Although the research presented in this paper has achieved good detection results in simulation experiments on actual datasets, there are still some problems in the whole algorithm that need to be improved. Firstly, the types of anomalies that can be identified in this article are not single, and they are not capable of identifying complex and diverse situations. Secondly, the algorithm is suitable only for the detection of abnormal behavior of low- and medium-density crowds, because when there are big crowds in the scene, severe occlusions will make the results of the number estimation algorithm inaccurate. Finally, the algorithm that detects the escape from the center does not detect more than three positions where anomalies may occur, and the algorithm is executed after detecting the occurrence of crowd abnormal events. How to optimize the calculation to achieve automatic and intelligent identification of the positions of crowd abnormalities that may occur is a key issue to be studied in the future.

Based on data mining, this study combined cloud computing image processing technology to identify realtime crime behavior. The abnormality detection object in this paper is a random group in ordinary public places, i.e., the people in the crowd are not unified and purposeful. The direction of movement of the crowd is irregular when there is no abnormality. At the same time, this study briefly illustrates that acceleration is an important motion feature of crowd anomaly detection. It also shows that the acceleration-based crowd anomaly detection algorithm is more reasonable than the traditional speed-based ones. Therefore, this paper uses the anomaly detection algorithm with improved acceleration characteristics to detect the abnormal escape behavior of the crowd. Firstly, the motion vector field is processed by block processing, and then the image is filtered to reduce the influence of noise. Next, the mean filtering is adopted, and then the algorithm is used to extract the foreground of the image sequence. This kind of operation not only facilitates the extraction of motion features, but also reduces the disadvantages of large computational complexity. The experimental research shows that the algorithm has high accuracy in identifying abnormal behavior and has high practical value, which can meet the accuracy and real-time requirements of the security system.

\section{DECLARATIONS}

\section{Authors' contributions}

Made substantial contributions to conception and design of the study and performed data analysis and interpretation: $\mathrm{Xu} \mathrm{Z}$

Performed data acquisition, as well as provided administrative, technical, and material support: Cheng C, Sugumaran V

\section{Availability of data and materials}

Not applicable. 


\section{Financial support and sponsorship}

This work is supported by National Key R\&D Program of China (No. 2018YFB1004605).

\section{Conflicts of interest}

All authors declared that there are no conflicts of interest.

\section{Ethical approval and consent to participate}

Not applicable.

\section{Consent for publication}

Not applicable.

\section{Copyright}

(c) The Author(s) 2020.

\section{REFERENCES}

1. Xie W. Research on big data processing model of multi objective genetic algorithm based on static bayesian game in cloud computing. J Comput Theor Nanosci 2016;13:9633-7.

2. Chen DY, Huang PC. Visual-based human crowds behavior analysis based on graphmodeling and matching. IEEE Sensors J 2013;13:2129-38.

3. Wu S, Wong HS, Yu Z. A Bayesian model for crowd escape behavior detection. IEEE T Circ Syst Vid 2014;24:85-98.

4. Biswas S, Babu RV. Anomaly detection in compressed H.264/AVC video. Multimed Tools Appl 2015;74:11099-115.

5. Cong Y, Yuan JS, Liu J. Abnormal event detection in crowded scenes using sparse representation. Pattern Recognit 2013;46:1851-64.

6. Li W, Mahadevan V, Vasconcelos N. Anomaly detection and localization in crowded scenes. IEEE T Pattern Anal 2013;36:18-32.

7. Song X, Wu M, Jermaine C. Conditional anomaly detection. IEEE Trans Knowl Data Eng 2007;19:631-45.

8. Kratz L, Nishino K. Tracking pedestrians using local spatio-temporal motion patterns in extremely crowded scenes. IEEE Trans Pattern Anal Mach Intell 2012;34:987-1002.

9. Liu W, Li J, Cho YB. A novel architecture for parallel multi-view HEVC decoder on mobile device. J Image Video Proc 2017;2017.

10. Benhajyoussef A, Ezzedine T, Bouallègue A. Gradient-based pre-processing for intra prediction in high efficiency video coding. J Image Video Proc 2017;2017.

11. Chu Y, Liu P. Some two-dimensional uncertain linguistic Heronian mean operators and their application in multiple-attribute decision making. Neural Comput Applic 2015;26:1461-80.

12. Mikaeil R, Haghshenas SS, Haghshenas SS, Ataei M. Performance prediction of circular saw machine using imperialist competitive algorithm and fuzzy clustering technique. Neural Comput Applic 2018;29:283-92.

13. Gupta BB, Badve OP. Taxonomy of DoS and DDoS attacks and desirable defense mechanism in a Cloud computing environment. Neural Comput Applic 2017;28:3655-82.

14. Chen L, Qu H, Zhao J, Chen B, Principe JC. Efficient and robust deep learning with Correntropy-induced loss function. Neural Comput Applic 2016;27:1019-31.

15. Tay KM, Jong CH, Lim CP. A clustering-based failure mode and effect analysis model and its application to the edible bird nest industry. Neural Comput Applic 2015;26:551-60.

16. Li G, Zhang L, Sun Y, Kong J. Towards the sEMG hand: internet of things sensors and haptic feedback application. Multimed Tools Appl 2019;78:29765-82.

17. Kowalczyk P, Sawicki D. Blink and wink detection as a control tool in multimodal interaction. Multimed Tools Appl 2019;78:13749-65.

18. Wang M, Chen W, Wang S, Liu J, Li X, et al. Answering why-not questions on semantic multimedia queries. Multimed Tools Appl 2018;77:3405-29.

19. Lei Y, Zhou X, Xie L. Emergency monitoring and disposal decision support system for sudden pollution accidents based on multimedia information system. Multimed Tools Appl 2019;78:11047-71.

20. Purificato E, Rinaldi AM. Multimedia and geographic data integration for cultural heritage information retrieval. Multimed Tools Appl 2018;77:27447-69. 